Supporting Information

\title{
Noncovalent stabilization of vesicular polyion complexes with chemically modified/single-stranded oligonucleotides and PEG- $b$-guanidinylated polypeptides for intracavity encapsulation of effector enzymes aimed at cooperative gene knockdown
}

Beob Soo Kim, ${ }^{1}$ Mitsuru Naito, ${ }^{2}$ Hiroyuki Chaya, ${ }^{1}$ Mao Hori, ${ }^{3}$ Kotaro Hayashi, ${ }^{4}$ Hyun Su Min, ${ }^{1}$ Yu Yi, ${ }^{1}$ Hyun Jin Kim, ${ }^{2}$ Tetsuya Nagata, ${ }^{5}$ Yasutaka Anraku, ${ }^{3}$ Akihiro Kishimura, ${ }^{6, *}$ Kazunori Kataoka, ${ }^{4,7}$ and Kanjiro Miyata ${ }^{1, *}$

${ }^{1}$ Department of Materials Engineering, Graduate School of Engineering, The University of Tokyo, 7-3-1 Hongo, Bunkyo-ku, Tokyo 113-8656, Japan

${ }^{2}$ Center for Disease Biology and Integrative Medicine, Graduate School of Medicine, The University of Tokyo, 7-3-1 Hongo, Bunkyo-ku, Tokyo 113-0033, Japan

${ }^{3}$ Department of Bioengineering, Graduate School of Engineering, The University of Tokyo, 7-31 Hongo, Bunkyo-ku, Tokyo 113-8656, Japan

${ }^{4}$ Innovation Center of NanoMedicne, Kawasaki Institute of Industrial Promotion, 3-25-14 Tonomachi, Kawasaki-ku, Kawasaki 210-0821, Japan

${ }^{5}$ Department of Neurology and Neurological Science, Graduate School, Tokyo Medical and Dental University, 1-5-45 Yushima, Bunkyo-ku, Tokyo 113-8519, Japan

${ }^{6}$ Department of Applied Chemistry, Faculty of Engineering, Kyushu University, 744 Moto-oka, Nishi-ku, Fukuoka 819-0395, Japan

${ }^{7}$ Institute for Future Initiatives, The University of Tokyo, 7-3-1 Hongo, Bunkyo-ku, Tokyo 1130033, Japan

\footnotetext{
* Corresponding authors

kishimura@mail.cstm.kyushu-u.ac.jp (A. Kishimura)

miyata@bmw.t.u-tokyo.ac.jp (K. Miyata)
} 
(a)

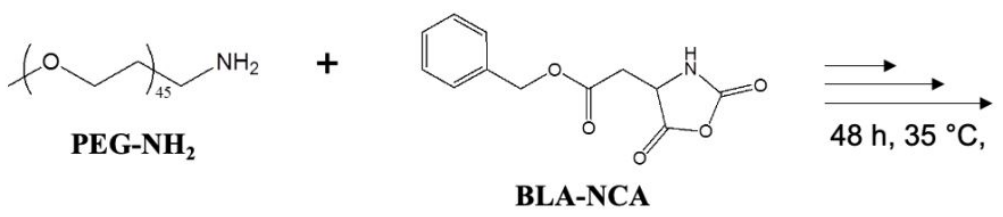

1,5-Diaminopentane

(DAP)

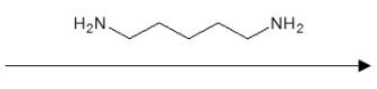

NMP, $5^{\circ} \mathrm{C}$,

(b)

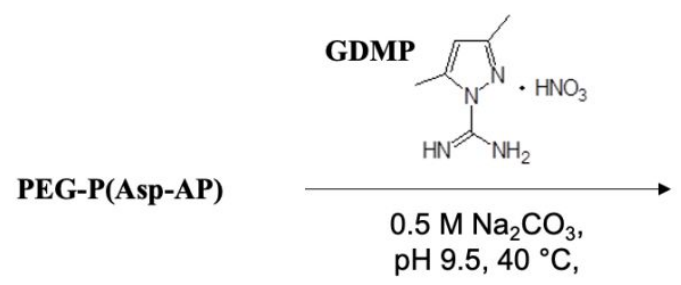

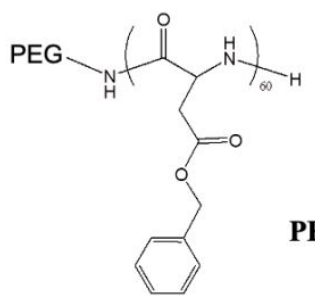

PEG-PBLA
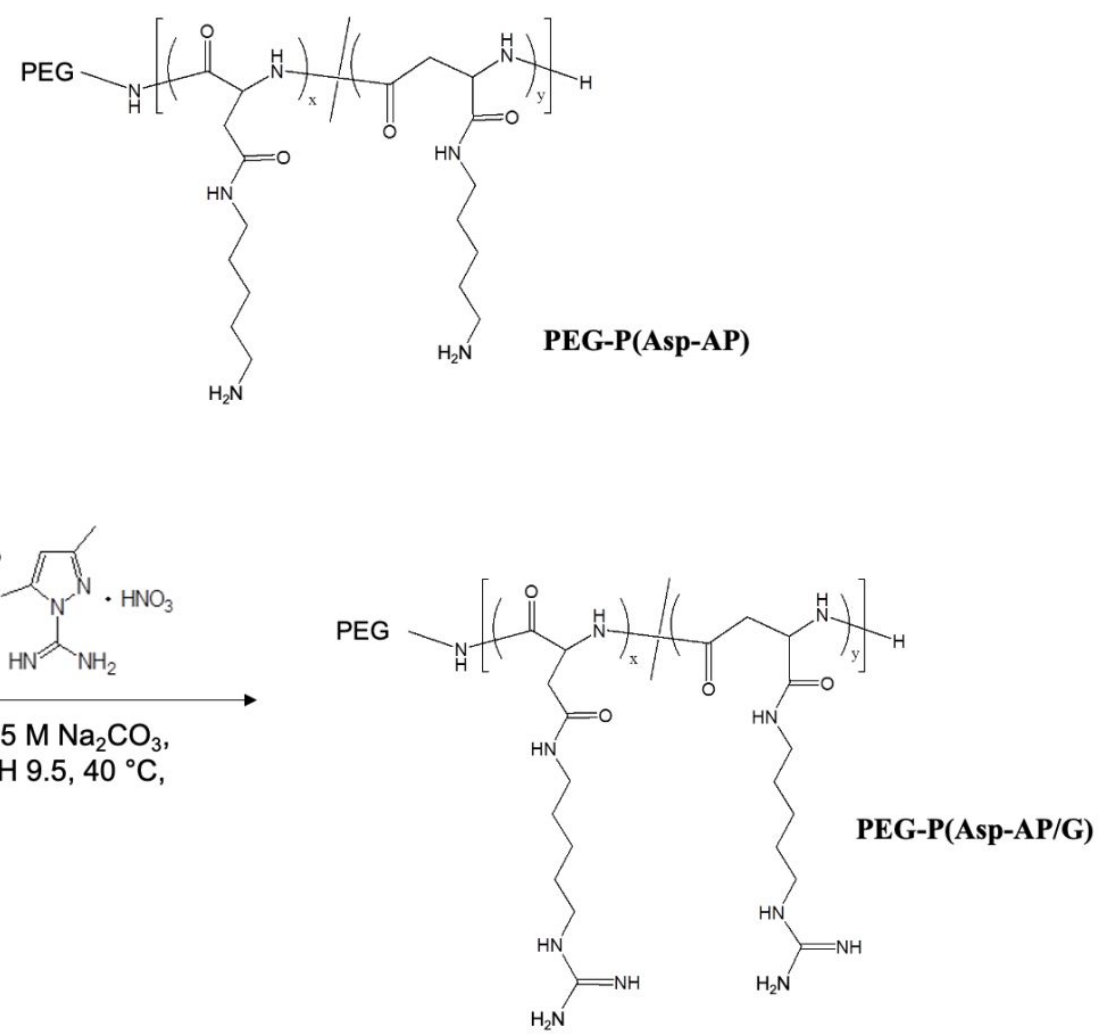

Scheme S1. Synthetic route of PEG-P(Asp-AP/G). (a) Ring-opening polymerization of BLA-NCA with PEG-NH${ }_{2}$ and aminolysis reaction of PEG-PBLA. (b) Guanidinylation of PEG-P(Asp-AP). 
Table S1. Structure and sequence information of a series of oligonucleotides used in this study.

\begin{tabular}{|c|c|c|}
\hline Target gene & Oligonucleotide & Structure $\left(5^{\prime} \text { to } 3^{\prime}\right)^{*}$ \\
\hline \multirow{4}{*}{ MALAT1 } & PO-DNA & atggaggtatgacatataatc \\
\hline & PS-DNA & $\mathrm{a}^{\wedge} \mathrm{t}^{\wedge} \mathrm{g}^{\wedge} \mathrm{g}^{\wedge} \mathrm{a}^{\wedge} \mathrm{g}^{\wedge} \mathrm{g}^{\wedge} \mathrm{t}^{\wedge} \mathrm{a}^{\wedge} \mathrm{t}^{\wedge} \mathrm{g}^{\wedge} \mathrm{a}^{\wedge} \mathrm{c}^{\wedge} \mathrm{a}^{\wedge} \mathrm{t}^{\wedge} \mathrm{a}^{\wedge} \mathrm{t}^{\wedge} \mathrm{a}^{\wedge} \mathrm{a}^{\wedge} \mathrm{t}^{\wedge} \mathrm{c}$ \\
\hline & PO-Gapmer & $A T G G$ aggtatgacatata $A T C$ \\
\hline & PS-Gapmer & $A^{\wedge} T^{\wedge} G^{\wedge} G^{\wedge} \mathrm{a}^{\wedge} \mathrm{g}^{\wedge} \mathrm{g}^{\wedge} \mathrm{t}^{\wedge} \mathrm{a}^{\wedge} \mathrm{t}^{\wedge} \mathrm{g}^{\wedge} \mathrm{a}^{\wedge} \mathrm{c}^{\wedge} \mathrm{a}^{\wedge} \mathrm{t}^{\wedge} \mathrm{a}^{\wedge} \mathrm{t}^{\wedge} \mathrm{a}^{\wedge} A^{\wedge} T^{\wedge} C$ \\
\hline \multirow{3}{*}{$T U G 1$} & PS-Gapmer & antisense: $T^{\wedge} G^{\wedge} A^{\wedge} A^{\wedge} \mathrm{t}^{\wedge} \mathrm{t}^{\wedge} \mathfrak{t}^{\wedge} \mathrm{c}^{\wedge} \mathrm{a}^{\wedge} \mathrm{a}^{\wedge} \mathrm{t}^{\wedge} \mathrm{c}^{\wedge} \mathrm{a}^{\wedge} \mathrm{t}^{\wedge} \mathrm{t}^{\wedge} \mathrm{t}^{\wedge} \mathrm{g}^{\wedge} \mathrm{a}^{\wedge} G^{\wedge} A^{\wedge} T$ \\
\hline & HDO & $\begin{array}{c}\text { antisense: } T^{\wedge} G^{\wedge} A^{\wedge} A^{\wedge} \mathrm{t}^{\wedge} \mathrm{t}^{\wedge} \mathrm{t}^{\wedge} \mathrm{c}^{\wedge} \mathrm{a}^{\wedge} \mathrm{a}^{\wedge} \mathrm{t}^{\wedge} \mathrm{c}^{\wedge} \mathrm{a}^{\wedge} \mathrm{t}^{\wedge} \mathrm{t}^{\wedge} \mathrm{t}^{\wedge} \mathrm{g}^{\wedge} \mathrm{a}^{\wedge} G^{\wedge} A^{\wedge} T \\
\text { sense: CUC AAA UGA UUG AAA UUC AUU }\end{array}$ \\
\hline & ssRNA & sense: CUC AAA UGA UUG AAA UUC AUU \\
\hline \multirow{2}{*}{ Control } & PS-Gapmer & $T^{\wedge} C^{\wedge} G^{\wedge} A^{\wedge} \mathrm{a}^{\wedge} \mathrm{g}^{\wedge} \mathrm{t}^{\wedge} \mathrm{a}^{\wedge} \mathrm{c}^{\wedge} \mathrm{t}^{\wedge} \mathrm{c}^{\wedge} \mathrm{a}^{\wedge} \mathrm{g}^{\wedge} \mathrm{c}^{\wedge} \mathrm{g}^{\wedge} \mathrm{t}^{\wedge} \mathrm{a}^{\wedge} \mathrm{a}^{\wedge} G^{\wedge} T^{\wedge} T$ \\
\hline & AF647-PS-Gapmer & $T^{\wedge} C^{\wedge} G^{\wedge} A^{\wedge} \mathrm{a}^{\wedge} \mathrm{g}^{\wedge} \mathrm{t}^{\wedge} \mathrm{a}^{\wedge} \mathrm{c}^{\wedge} \mathrm{t}^{\wedge} \mathrm{c}^{\wedge} \mathrm{a}^{\wedge} \mathrm{g}^{\wedge} \mathrm{c}^{\wedge} \mathrm{g}^{\wedge} \mathrm{t}^{\wedge} \mathrm{a}^{\wedge} \mathrm{a}^{\wedge} G^{\wedge} T^{\wedge} T-\mathrm{AF} 647$ \\
\hline
\end{tabular}


Table S2. Size and PDI of PICs prepared from varying oligonucleotides in $10 \mathrm{mM}$ HEPES buffer (pH 7.3).

\begin{tabular}{|c|c|c|c|c|}
\hline PEG- $b$-polypeptide & \multicolumn{2}{|c|}{ Oligonucleotide } & $D_{\mathrm{H}}(\mathrm{nm})^{*}$ & PDI* \\
\hline \multirow{7}{*}{ PEG-P(Asp-AP/G80) } & \multirow{4}{*}{ MALAT1 } & PO-DNA & $116 \pm 10$ & $0.17 \pm 0.03$ \\
\hline & & PS-DNA & $87 \pm 2$ & $0.24 \pm 0.05$ \\
\hline & & PO-Gapmer & $113 \pm 5$ & $0.25 \pm 0.04$ \\
\hline & & PS-Gapmer & $103 \pm 3$ & $0.10 \pm 0.03$ \\
\hline & \multirow{2}{*}{$T U G 1$} & PS-Gapmer & $102 \pm 4$ & $0.12 \pm 0.02$ \\
\hline & & HDO & $103 \pm 5$ & $0.23 \pm 0.03$ \\
\hline & $M A L A T 1 / T U G 1$ & PS-Gapmer / ssRNA & $95 \pm 6$ & $0.19 \pm 0.04$ \\
\hline
\end{tabular}

*Oligonucleotide concentration: $10 \mu \mathrm{M}$, mean $\pm \mathrm{SD}(n=4)$.

Table S3. Relative SLI, size, and PDI of PICs prepared from PEG-P(Asp-AP/G) with varying guanidinylation degrees in 10 mM HEPES buffer ( $\mathrm{pH} 7.3)$.

\begin{tabular}{cccccc}
\hline PEG- $b$-polypeptide & Oligonucleotide & $\begin{array}{c}\text { Relative } \\
\text { SLI }^{\mathrm{a}, \mathrm{b}}\end{array}$ & $D_{\mathrm{H}}(\mathrm{nm})^{\mathrm{b}}$ & PDI $^{\mathrm{b}}$ & $\begin{array}{c}\text { Zeta-potential }^{\mathrm{b}} \\
(\mathrm{mV})\end{array}$ \\
\hline PEG-P(Asp-AP) & & $29 \pm 9$ & $370 \pm 97$ & $0.42 \pm 0.19$ & $0 \pm 2$ \\
PEG-P(Asp-AP/G20) & MALAT1- & $262 \pm 22$ & $301 \pm 94$ & $0.41 \pm 0.14$ & $-2 \pm 3$ \\
PEG-P(Asp-AP/G50) & PS-Gapmer & $443 \pm 68$ & $96 \pm 5$ & $0.18 \pm 0.03$ & $-16 \pm 3$ \\
PEG-P(Asp-AP/G80) & & $970 \pm 88$ & $103 \pm 3$ & $0.10 \pm 0.03$ & $-18 \pm 2$ \\
\hline
\end{tabular}

${ }^{a}$ [Derived count rate of PICs]/[derived count rate of PEG- $b$-polypeptide without oligonucleotide]

${ }^{\mathrm{b}}$ Oligonucleotide concentration: $10 \mu \mathrm{M}$, mean $\pm \mathrm{SD}(n=4)$. 


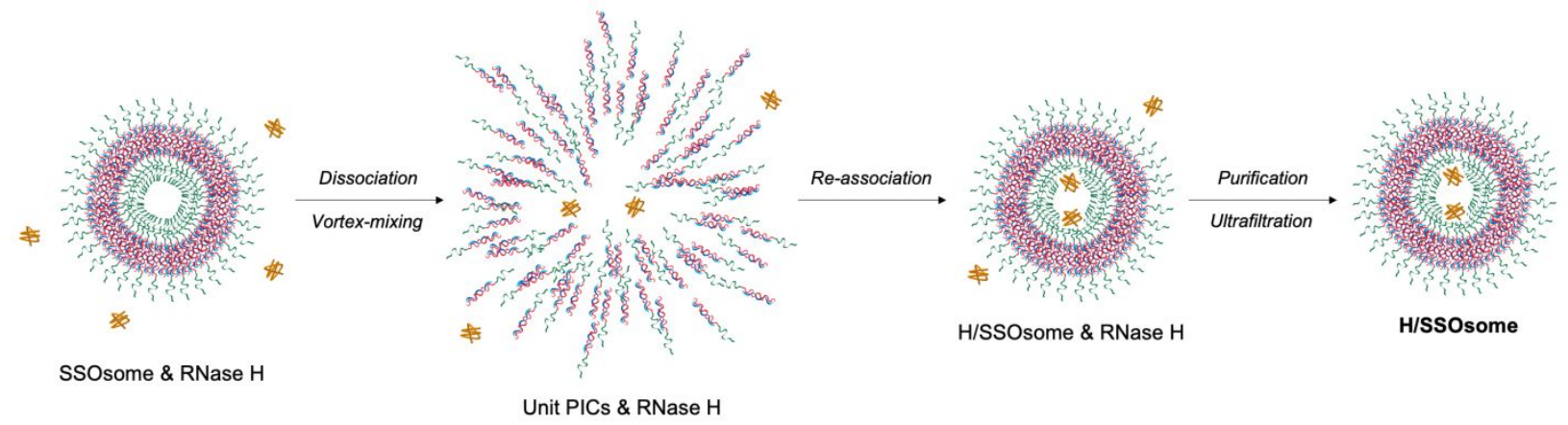

Figure S1. Schematic illustration of the physical encapsulation of enzymes (or RNase H) within a vesicular PIC (or SSOsome) through the dissociation and re-association process between the vesicular PIC and unit PICs. 


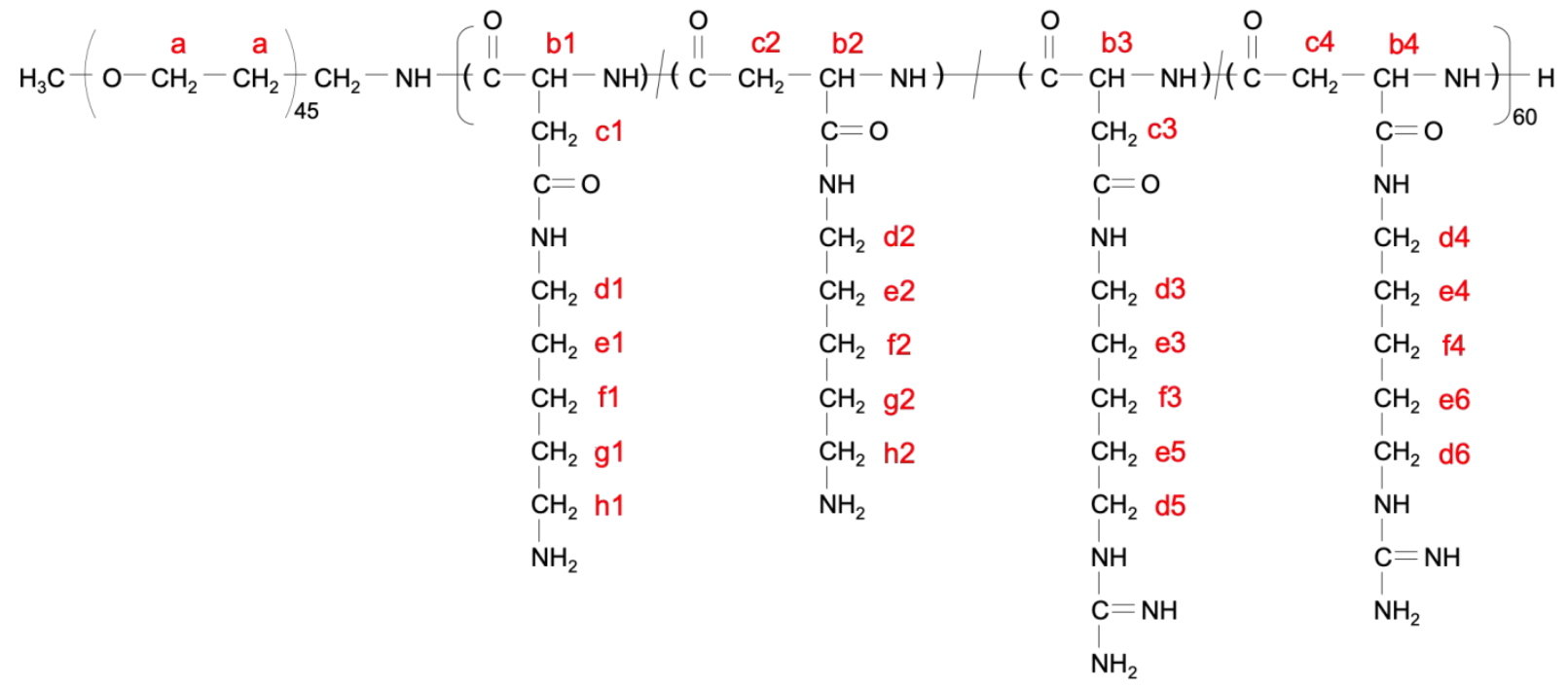

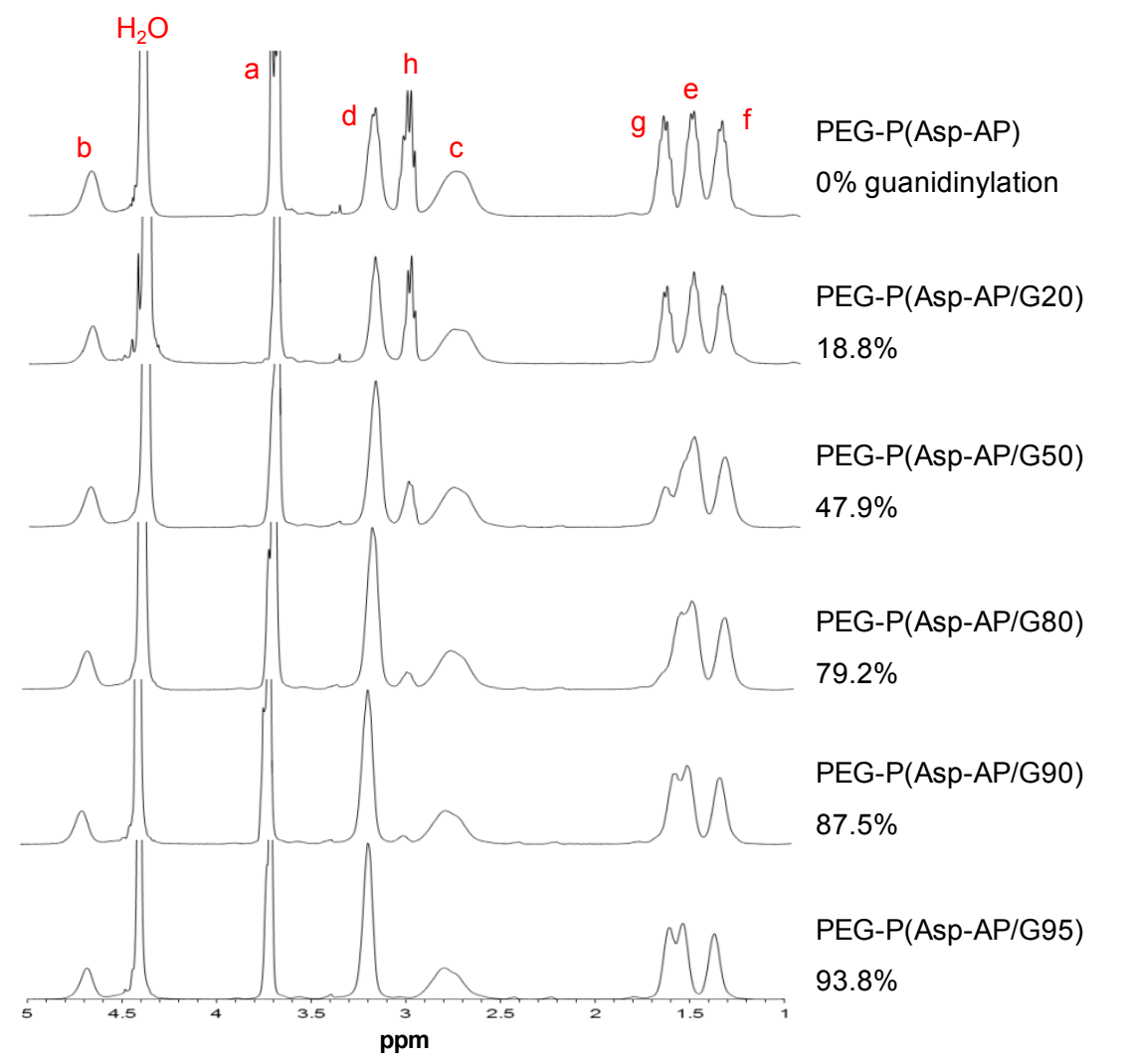

Figure S2. ${ }^{1} \mathrm{H}$ NMR spectra of PEG-P(Asp-AP) and PEG-P(Asp-AP/G)s (polymer concentration: $6 \mathrm{mg} / \mathrm{mL}$, solvent: $\mathrm{D}_{2} \mathrm{O}$, temperature: $80^{\circ} \mathrm{C}$ ). 


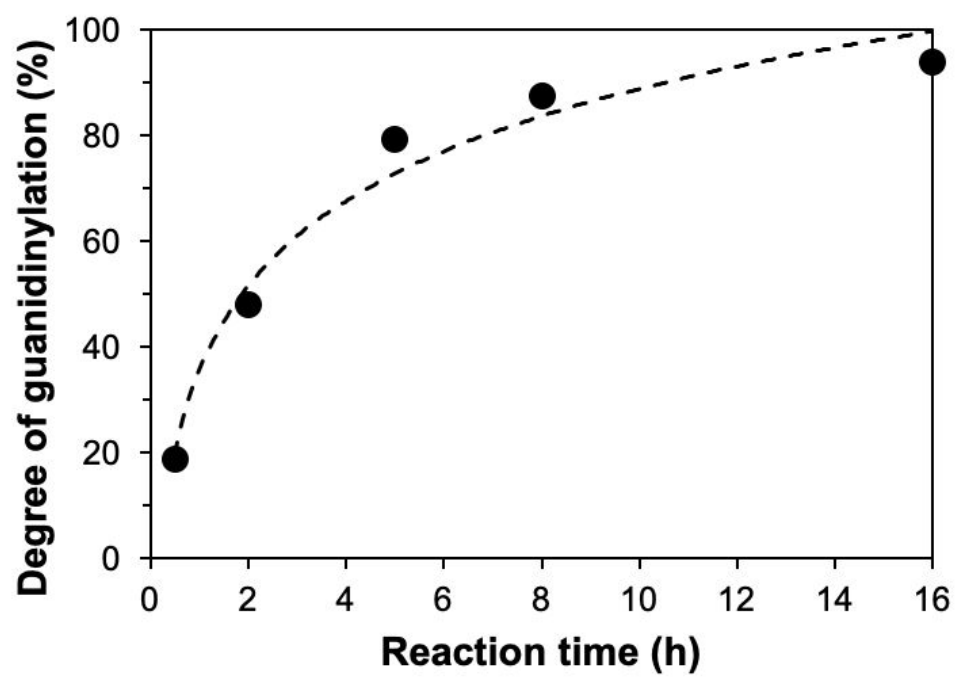

Figure S3. Time-dependent rate of PEG-P(Asp-AP) guanidinylation.

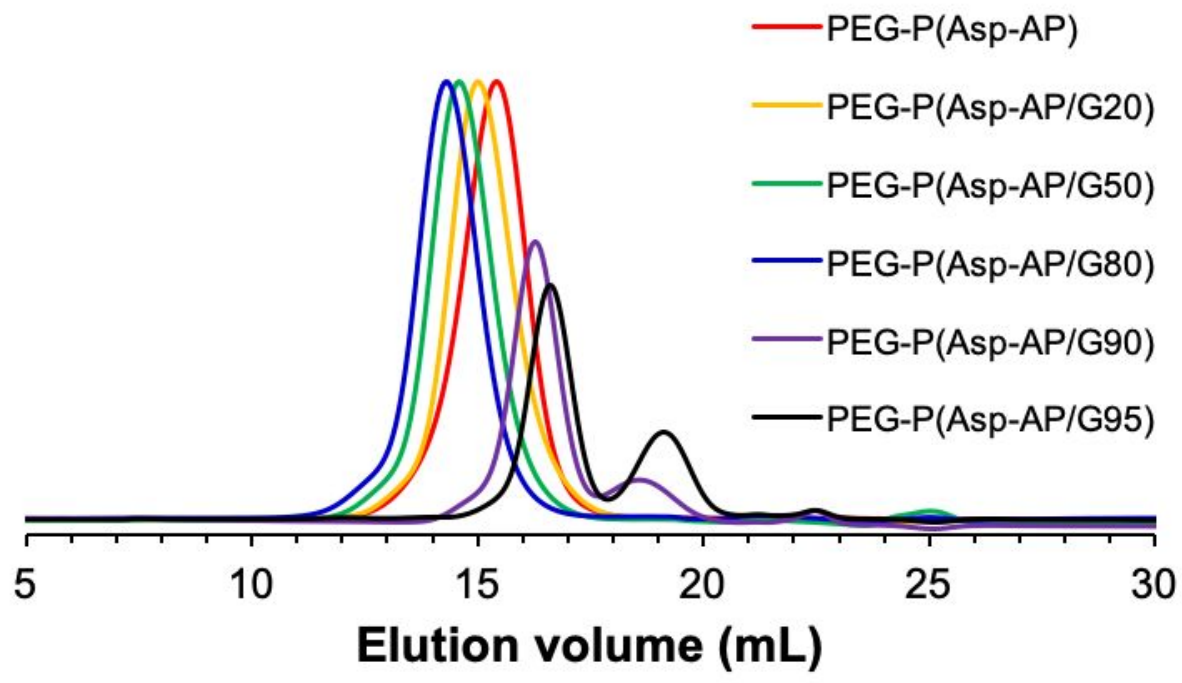

Figure S4. SEC profiles of PEG-P(Asp-AP) and PEG-P(Asp-AP/G)s (eluent: $10 \mathrm{mM} \mathrm{AcOH,} \mathrm{pH}$ 4.6, $500 \mathrm{mM} \mathrm{NaCl}, 0.75 \mathrm{~mL} / \mathrm{min}$, room temperature). 


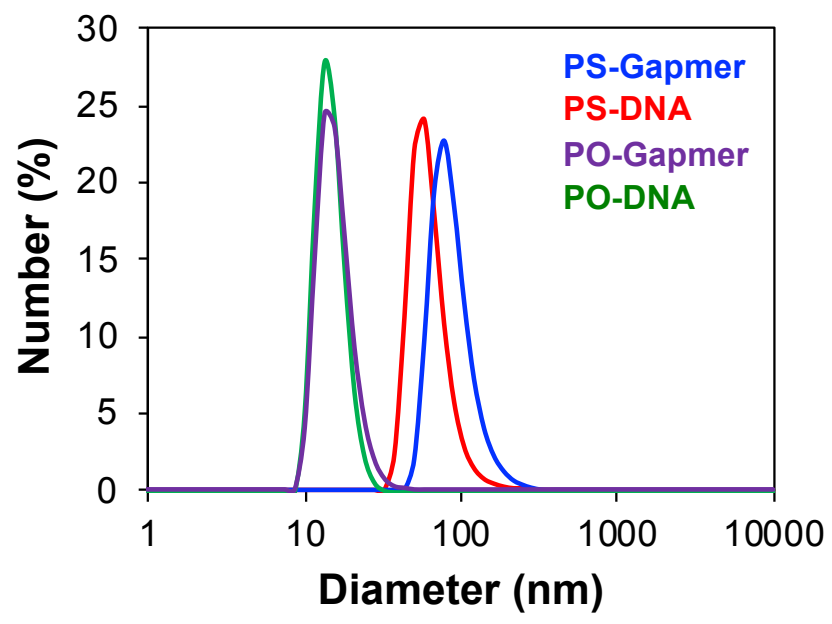

Figure S5. Number-weighted size distribution histograms of PICs prepared from PEG-P(AspAP/G) and PO-DNA, PO-Gapmer, PS-DNA, or PS-Gapmer. The samples were incubated at 0.1 $\mathrm{mg} / \mathrm{mL}$ in $10 \mathrm{mM}$ HEPES buffer (pH 7.3) containing $150 \mathrm{mM} \mathrm{NaCl}$ at $25^{\circ} \mathrm{C}$ for $48 \mathrm{~h}$.

(a)

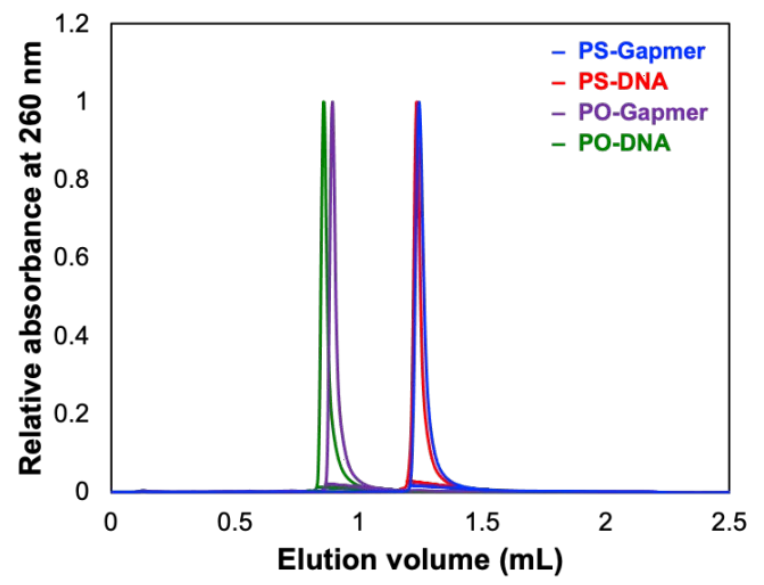

(b)

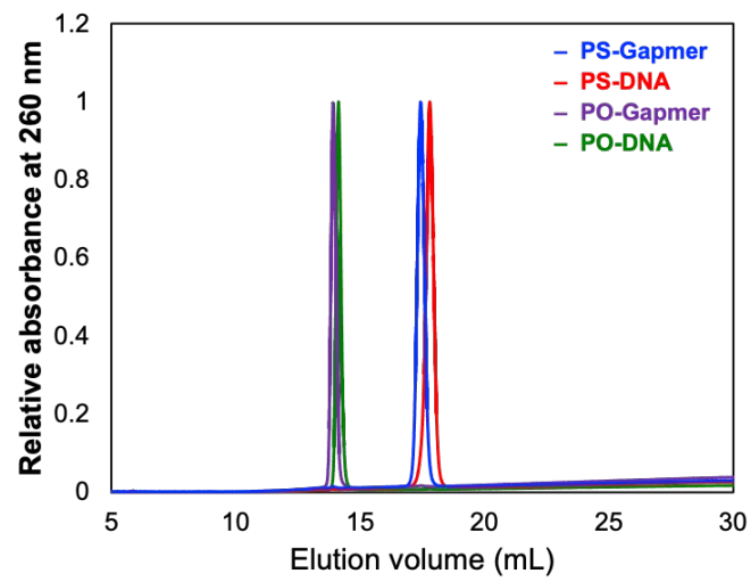

Figure S6. Chromatographic analyses of a series of SSOs with/without the PS backbone and LNA-Gapmer. (a) Reverse phase liquid chromatography (eluent A: $8 \mathrm{mM}$ triethylamine, $100 \mathrm{mM}$ HFIP, $\mathrm{H}_{2} \mathrm{O}$, eluent B: methanol, gradient: $10-25 \% \mathrm{~B}, 0.5 \mathrm{~mL} / \mathrm{min}, 60^{\circ} \mathrm{C}$ ). (b) Ion exchange liquid chromatography (eluent $\mathrm{C}$ : $0.1 \mathrm{M}$ sodium hydroxide, eluent $\mathrm{D}$ : $0.1 \mathrm{M}$ sodium hydroxide, $1 \mathrm{M}$ sodium perchlorate, gradient: $5-100 \% \mathrm{D}, 2.0 \mathrm{~mL} / \mathrm{min}$, room temperature). 


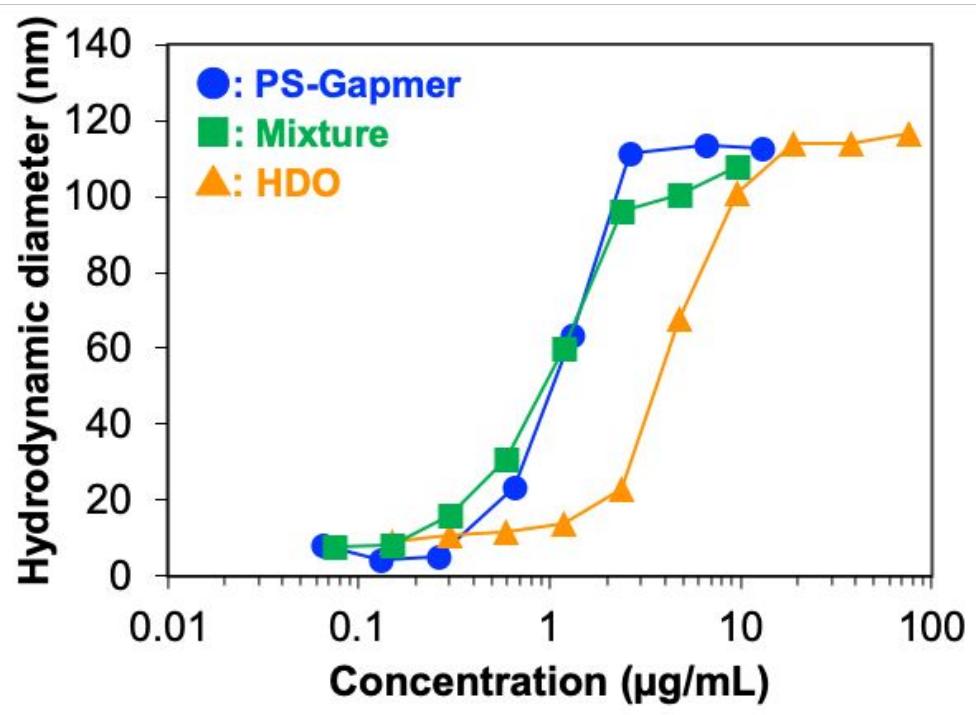

Figure S7. Concentration-dependent change in the size of PICs in 10 mM HEPES buffer (pH 7.3) without $\mathrm{NaCl}$. PIC samples were prepared at varying concentrations of PEG-P(Asp-AP/G80) and AF647-PS-Gapmer, AF647-HDO, or AF647-PS-Gapmer/ssRNA (Mixture). The hydrodynamic diameters were determined using FCS analysis.

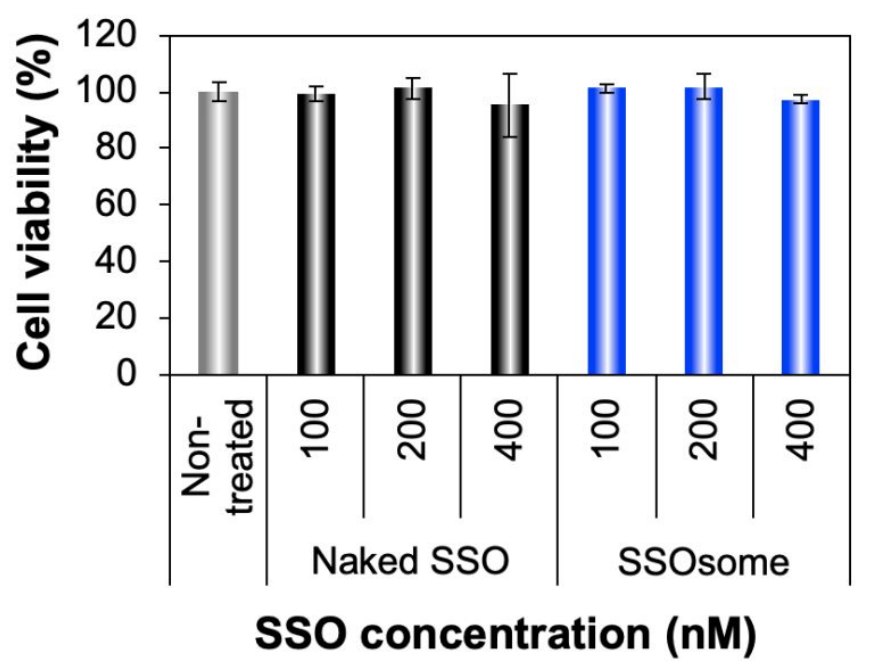

Figure S8. Viability of A549 cells after 48-h incubation with naked Control-SSO and ControlSSOsomes. Results are expressed as mean $\pm \mathrm{SD}(n=4)$. 




Figure S9. TEM image of H/SSOsome. Scale bar represents $100 \mathrm{~nm}$.

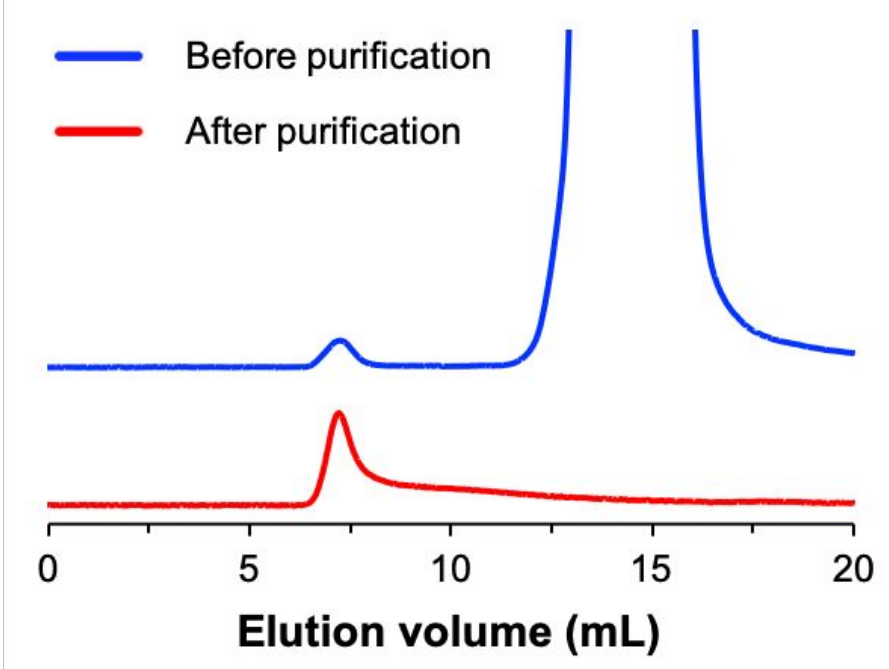

Figure S10. SEC profiles of H/SSOsome before (blue) and after (red) purification (eluent: 10 $\mathrm{mM}$ PB, $\mathrm{pH} 7.4,150 \mathrm{mM} \mathrm{NaCl}, 0.5 \mathrm{~mL} / \mathrm{min}$, and room temperature). 


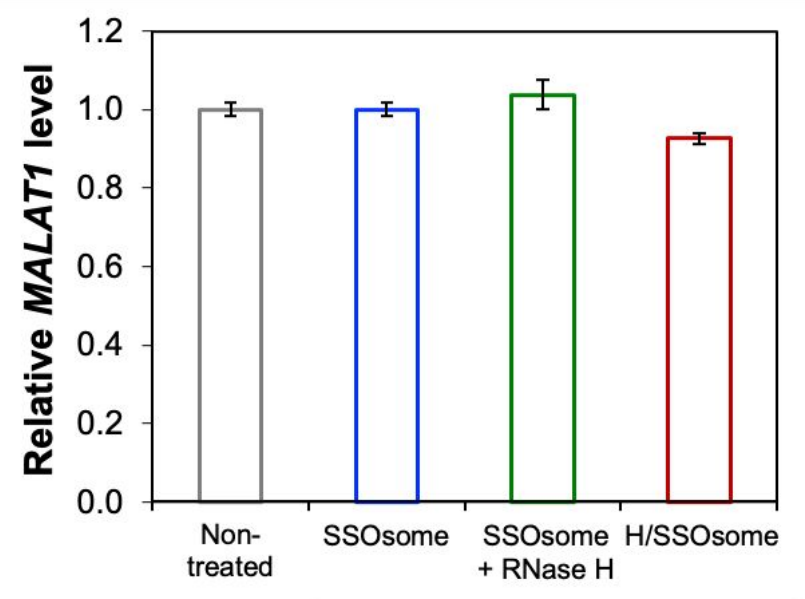

Figure S11. Gene knockdown efficiency determined using qRT-PCR. A549 cells were incubated with Control-SSOsome, a mixture of Control-SSOsome and free RNase H (SSOsome + RNase $\mathrm{H}$ ), or Control-H/SSOsome at $100 \mathrm{nM} \mathrm{Control-SSO}$ and at $100 \mathrm{pM} \mathrm{RNase} \mathrm{H} \mathrm{for} 48 \mathrm{~h}$. Results are expressed as mean $\pm \mathrm{SD}(n=4)$.

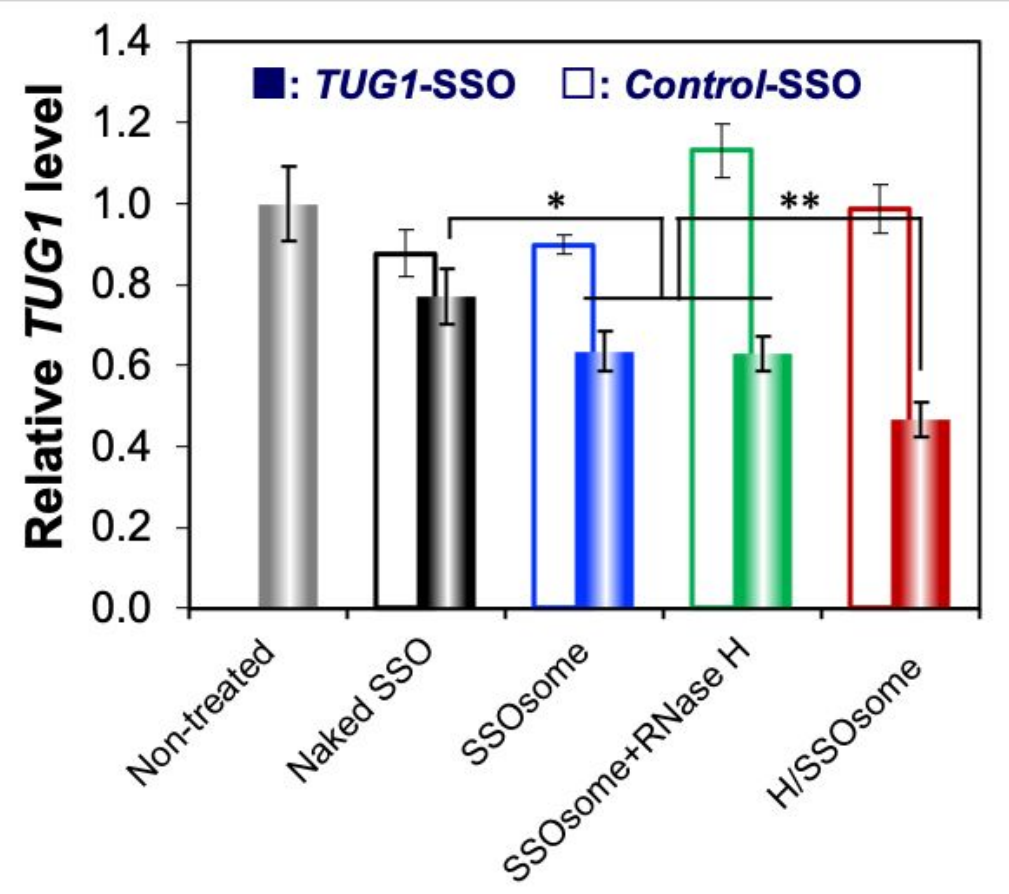

Figure S12. Gene knockdown efficiency determined using qRT-PCR. Panc1 cells were incubated with naked SSO, SSOsome, a mixture of SSOsome and free RNase H (SSOsome + RNase H), or $\mathrm{H} / \mathrm{SSOsome}$ at $100 \mathrm{nM} \mathrm{TUGl}$ (or Control)-SSO and at $100 \mathrm{pM}$ RNase H for $48 \mathrm{~h}$. Results are expressed as mean $\pm \mathrm{SD}(n=4) .{ }^{*} p<0.05$ and ${ }^{* *} p<0.01$. 